\title{
Designing Modelling Methods: The Learn PAd Scenario
}

\author{
FOR WORKSHOP SESSION " Conceptual \\ Modelling and Semantics for Value-based Knowledge Transfer" \\ Nesat EFENDIOGLU, Robert WOITSCH, Mehmet ALBAYRAK \\ ${ }^{1}$ BOC Asset Management GmbH, 1040 Vienna, Operngasse $20 B$ \\ Tel: +43-1-905 10 56, Fax: +43-1-905 10563007
}

Email:\{firstname.lastname \}@boc-eu.com:

\begin{abstract}
Modelling methods are commodity to enable a conceptual knowledge base for smart and intelligent software. As programmes become more complex and requirements for such conceptual or semantic knowledge bases raises, the current development approaches reach their limits. The project Learn PAd applies a modeldriven approach for technology enhanced learning and knowledge management in the domain of public administration and elaborates a new way of designing and consequently in developing modelling methods. Based on the research of the Open Model Laboratory (OMiLAB) community, Learn PAd implemented a design and development tool for modelling methods. The result had been tested in the project, is currently used and improved in other projects and available for usage and contributions on the ADOxx.org portal.
\end{abstract}

\section{Introduction -}

Nowadays the public administrators are struggling while adapting rapid changes in their working environment, (e.g changes in regulations, law, society and evolution of technology). The most promising solution for that challenge is probably transformation of public administration organizations into learning organizations [1] where they can create and use their knowledge assets through the collaborative intelligence [2] [3] [4] [5] [6] For the transformation the EU project Learn PAd [7] proposes a process-driven-knowledge management approach based on conceptual and semantic models.

The Learn PAd EU-Project aims to support public administration employee's so-called "civil servants" five concrete scenarios [8]; (1) Individual Training (2) Organizational Evolution, (3) Support and Reflection, (4) Process Optimization and Improvement, (5) Citizen Transparency

The project targets to provide a collaborative learning platform for civil servants, where they will be engaged in a collaborative learning and assessment experience. The platform is centred on the business process, which is graphically represented and continuously evolved within the public administration and their surrounding context. The platform complements the graphical representation of conceptual models with descriptive contents producing enriched and machine-process able models.

Realization of Learn PAd e-learning platform for civil servants is based on four pillars; (1) A new concept of model based e-learning, (2) open and collaborative e-learning content management, (3) automatic, learner-specific and (4) collaborative content quality assessment and simulation-based learning and testing.

Given that innovation of Learn PAd is based on a model-driven approach, it proposes a new modelling method, which will be focused in the rest of this paper. 
The development of new modelling method for such a complex domain with ambitious objectives brings following challenges:

- Modelling method has to consider the following aspects; (a) business processes, (b) motivation, (c) organizational structure and roles, (d) knowledge resources, (e) learning goals, (f) performance measurements.

- Existing technologies that can realize the solution need to be considered in order to enable a flexible deployment into the legacy Application. (e.g ADOxx, XWiki)

- The modelling language shall be flexible enough to be implemented on different platforms hence the consortium focuses on a new way of defining the meta-model. Three different abstraction layer are considered (a) Conceptual Meta-model (CMM) specifies formal semantics of concepts that are the abstraction of aspects relevant for eLearning within Public Administration, (b) Platform Independent Meta-model (PIMM) )) that elaborates the aspects with considering application scenarios, requirements in manner of functional, non-functional requirements, but without considering any specific meta-modelling platform or technology, (c) Platform Specific Meta- model (PSMM) elaborates -addition to PIMM- the technology that is utilized to implement meta-model, hence it considers platform specific concepts and design constraints.

- Beside abovementioned challenges, another challenge is caused by collaboration for development of the modelling method. Given that the multiple experts from different domains (from software engineering and modelling, business process modelling and analysis, knowledge and learning management, and public administrations shall collaborate to develop the modelling method), the development approach should be appropriate to varying background of those experts.

In order to address aforementioned challenges this paper proposes: (a) investigation of appropriate modelling method development framework, (b) appropriate conceptualization process and especially (c) introduction and evaluation of a modelling method design environment that should support conceptualization process with considering the above mentioned challenges.

Correspondingly first of all, we introduced the selected modelling method development framework, secondly we introduce requirement collection and analysis method that we have utilized to discover the aspects and corresponding functional and non-functional requirements that need to be considered in the meta-model, and the Modelling Method Design Environment and finally we present evaluation results of the design environment that have been collected during conceptualization of Learn PAd meta model, particularly concentrating on create, design and formalization phases

\section{Conceptualization of Meta Model}

In the section we briefly elaborated on the challenges such as investigation of varying aspects in settings of e-learning scenarios particularly in public administration domain as well a specific knowledge process to be executed, a cooperation of different stakeholders with varying expertise and perspective is required during the conceptualization of Learn Pad Meta-Model. We utilized (1) Generic Modelling Method Specification Framework, which guides the modelling method engineers during the development of domain specific modelling languages (hence meta-models of modelling languages) and mapping appropriate functionalities, also (2) conceptualization process adapted from OMILAB Life Cycle, which defines clearly the conceptualization phases of a modelling method.

\subsection{The Metamodeling Approach and Generic Modelling Method Specification Framework}

The Generic Modelling Method Specification Framework (GMMSF) is introduced by Karagiannis and Kühn in [8] [9]. The GMMSF is a modelling method specification 
Framework which has been successfully applied in more than 20 EU Framework Program projects, more than 30 various research projects and in dev elopement of different commercial model-driven-management tools (e.g. ADONIS $^{\circledR}$, ADOlog $^{\circledR}, \quad$ ADOit $^{\circledR}$, ADOscore $^{\circledR}$, PROMOTE $^{\circledR}$, ADVISOR $^{\circledR}$, etc.)

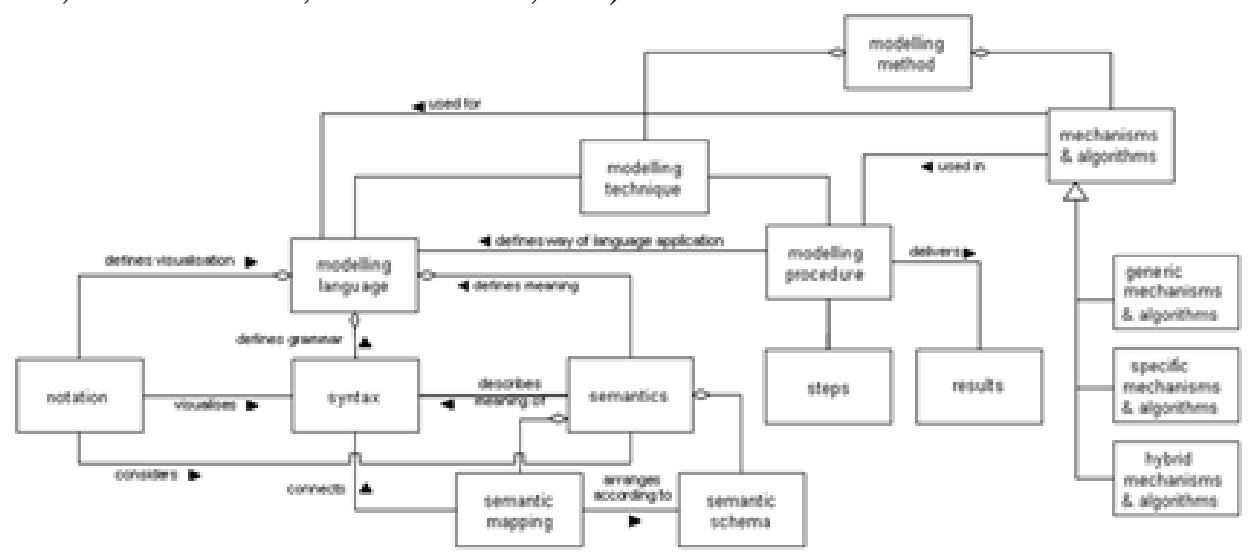

Figure 1 Generic Modelling Method Specification Framework [8] [9]

As depicted in Figure 1 the building blocks of a modelling method include: (1) the modelling language introducing modelling concepts pre-defined according their semantic, their syntax and their graphical notation, (2) the modelling procedure which defines the stepwise usage of the modelling language and may not be always available and (3) generic and domain specific mechanisms and algorithms enabling the computer-based processing of models.

\subsection{Conceptualization Process}

The Figure 2 depicts the modelling method conceptualization process based on OMILAB LifeCycle [11], which is utilized during the conceptualization of Learn PAd Modelling Method. The OMILAB Lifecycle consists of five phases; (1) Create Phase, where the system under study, application scenarios and their requirements are investigated, (2) Design Phase, where the modelling language with its syntax, semantic and notation in terms of meta-models is specified, mechanisms and algorithms are specified, (3) Formalization Phase, where the specification of modelling language is well-formalized to ensure a possible implementation into software. (4) Development Phase, where the specified modelling method is implemented on a meta-modelling environment, (5) Deployment phase, where the implementation is packed in form of standalone application and offered to use of end-users.

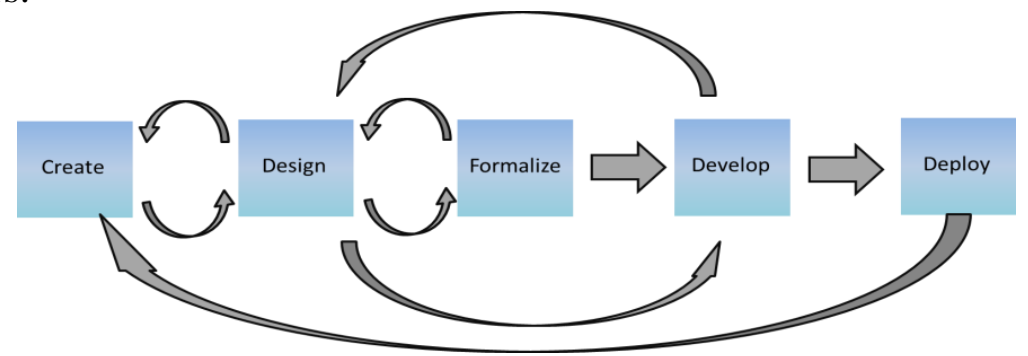

Figure 2 Modelling Method Conceptualization Process (based on OMILAB Life Cycle [11]]

The OMILAB Lifecycle considers the conceptualization process in a sequence where the feedback about modelling method provided after deployment to the create phase for possible improvements. Having the stakeholder with varying knowledge background and perspective involve in conceptualization of the modelling method, and on the other hand stakeholders at end-user side with different objectives, we adapted the OMILAB LifeCylce 
and feedback channels between create and design, in order to prove, if the designed modelling language covers identified application scenarios and considers the identified requirements, between design and formalize to ensure formal approval of modelling language, as well as between design and development phases in order to improve modelling language in earlier stages before it is released and deployed.

\section{Modelling Method Design Environment}

The Modelling Method Design Environment (MMDE) has to support the conceptualization process of Learn PAd Modelling Method. The following requirements have been considered:

- Definition of separated meta-models should be possible.

- Definition of dependencies among meta-models and their interaction should be possible.

- The design environment should enables domain experts to participates the specification of the syntax, semantics and the modelling method

- Tracing the modelling method requirements.

There are many meta-modelling platforms available in the market that would support the development and deployment phases of a modelling method, such as Eclipse with EMF [12], ADOxx [13], MetaEdit+ [14], AToM ${ }^{3}$ [15], GME [16]. There are also available tools such as MOF, OWL, UML, XML and their corresponding design platforms, which could support the experts during the create and design phases.

Those tools have respectable capabilities, and potentially benefit from a platform independent meta model design environment providing features such as:

- Intuitive enough to be used without expert knowledge

- Specification of weaving among concepts, with another words in relations among concepts in separated meta-models

- Navigational Elements in order to simplify readability of complex meta-models

- Specification of graphical notation of modelling language

- Definition of requirements and tracing if they are considered in the implementation or not

We took a subset of UML and extend the modelling language with required concepts and model types, and developed modelling language "Modeling Method Design Language" and implemented it into a design environment so-called "Modelling Method Design Environment" available free to download with samples in [17].

In following sections we introduce the solutions differentiating solutions to support the Create Phase and solutions to Support Design Phase.

\subsection{Solutions to Support Create Phase}

On the basis that (1) Learn PAd solution is a socio-technical system (2) Learn PAd proposes a process-driven approach based on conceptual and semantic models for transforming public administration organizations into learning organizations and on the other hand (3) the Learn PAd solution is realized on existing technologies; and finally (4) targeting to support public administrators in varying scenarios with functionalities defined in wide spectrum, we collect and analyse the requirements from four different dimension [7]; (a) Learn PAd Paradigm describes social-technical eco-system of LearnPAd, (b) Learn PAd Technology describes all technical aspects and core components of Learn PAd, (c) Learn PAd (modelling-) Language describes the bridge between Learn PAd Paradigm and Technology by introducing conceptual and semantic modelling and obiviously (d) Learn PAd Usage describes application of Learn PAd platform into the organizations considering 
varying organization-cultures and -eco-systems. In order to collect and analyse the requirement, a collaborative bottom-up collection process had been performed using the well known Volere [10]

In this work, we took under consideration the requirements that have been grouped under the dimension Learn PAd language, which are relevant to the conceptualization of the meta-model of Learn PAd modelling method and its formalization in form of rapid prototypes. Hence during the Create Phase, we strive to provide solutions enables description and status tracing of the requirements regarding to modelling method.

To be able to describe and trace the requirements we specify another model type called "Requirements Model Type"

Requirements Model Type: (as depicted in Figure 3 ) allows describing the requirements, specifying their status as well as dependencies among them. The described requirements in this model type can be referenced to the all concepts in the meta-model in class level, graphical notation definitions, model types in modelling stack and functionalities described in mechanisms \& algorithms model type.

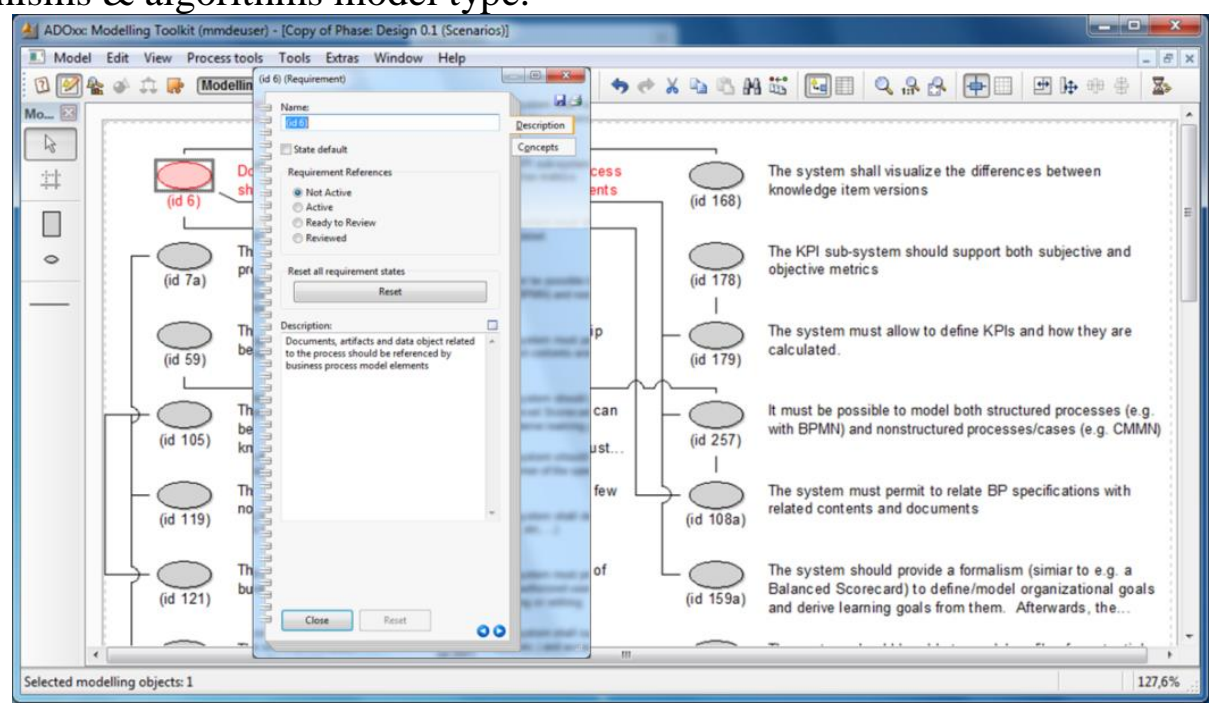

Figure 3Sample Requirement Model and Describtion of Requirements

As depicted in Figure 4 depending on the status of those concepts the status of the requirements will be updated accordingly. It is enough, when the user clicks of menu item "Update Requirements Status", the design environment will check if the requirement has been considered in any concept within the modelling method. If yes, the design environment will update the status of requirement accordingly and list the concepts where the requirement has been considered in the notebook of the requirement.

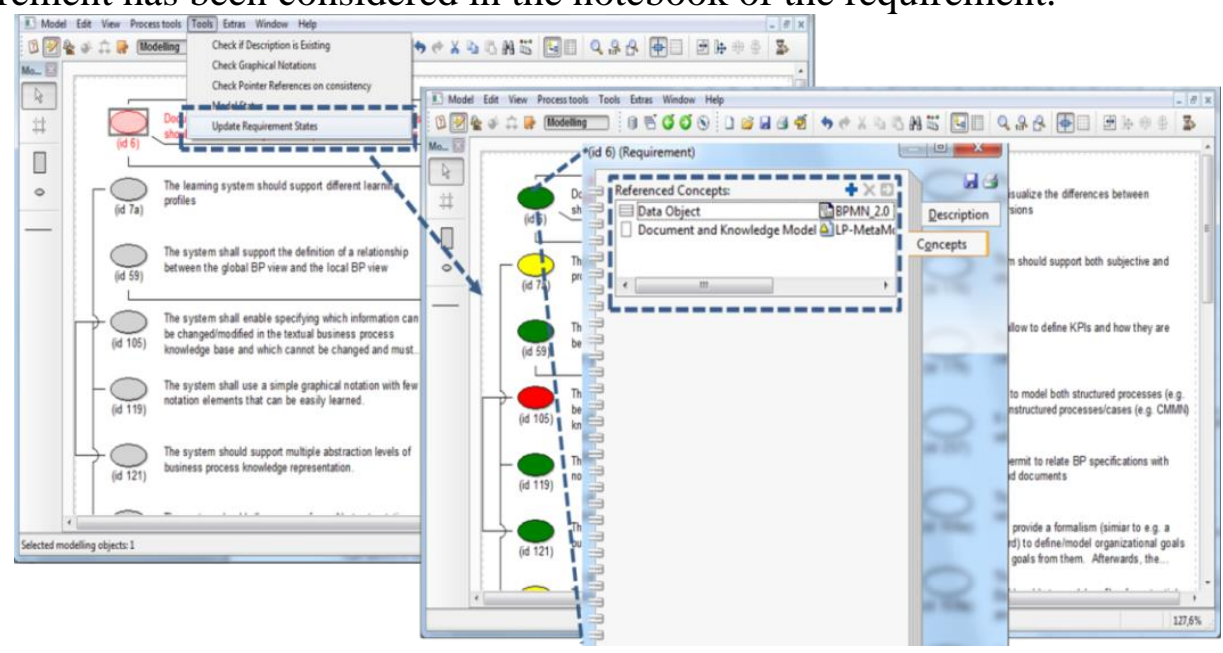

Figure 4 After automatically Updating Status of Requirements 
The design environment differentiates the status of requirement with colour codes; Grey, if the requirement not considered yet, Red if the requirement not considered and dismissed, Yellow, if the requirement is in consideration and Green if the requirement considered and reviewed.

\subsection{Design Phase}

Since the design phase the phase, where the specification of concrete meta-model is being made, where the more intensive collaboration is needed, the most of supportive solutions of the Modelling Method Design Environment target the actions in this phase. The following solutions are offered by the design environment

First of all to ease the readability and navigation we specified a new model type "MeatModel- Stack

Meta-Model-Stack Model Type: in order to model meta-models considering them as different model type that targets different aspects, we propose the Meta-Model-Stack Model Type. This model type allows description of hierarchy and dependencies among metamodels on a higher-level, without giving details about each meta-model in form of classes, attributes or cardinalities etc. This model type consists of navigational modelling concepts which enable aggregation of meta-models, definition of their scope and relations among them. On the other hand this model type allows to trace development status of each metamodel, with another words status of each model type.

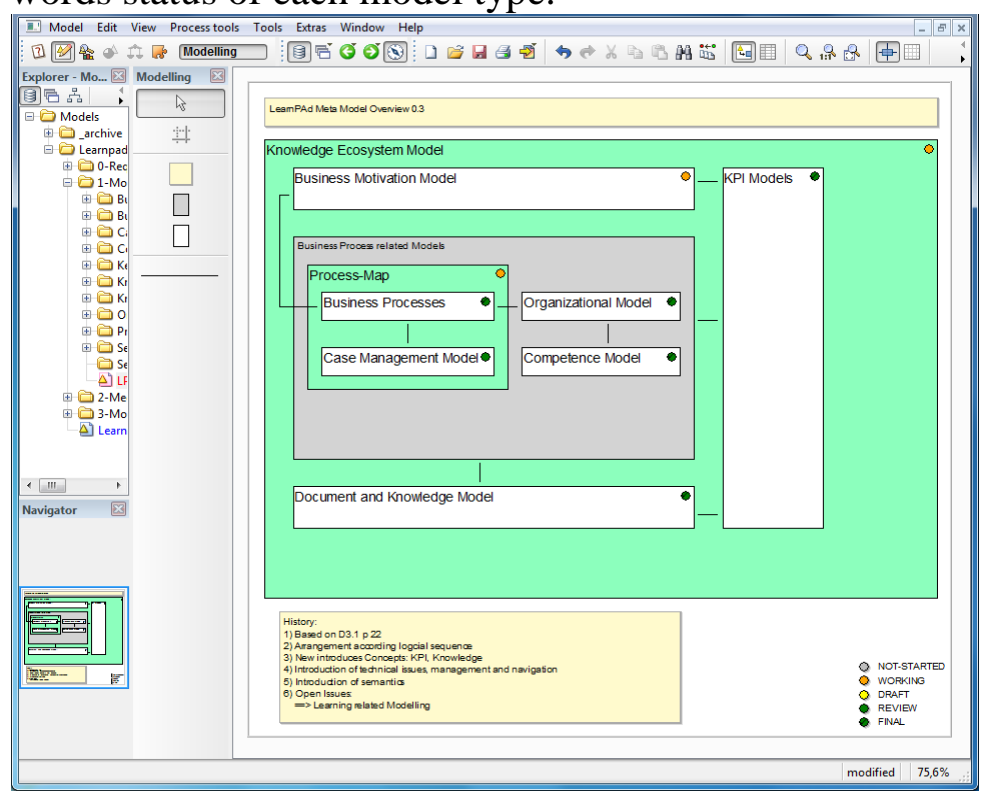

Figure 5Meta-Model Stack Model Type Sample

In order to specify the meta-models in any of three abstraction, we specified the model type "Meta-model Classes Model Type" which is following MOF standards and consists of sub-set of UML. Most important proposal in this model type is "Pointer" modelling construct in order to specify weaving among the concepts in separated meta-models.

Meta Model Classes Model Type, this model types enables specification of different meta models with their semantic primitives; classes, attributes, associations, and with specific relation "Pointer" in order enable weaving or specify dependencies among concepts in different model types 


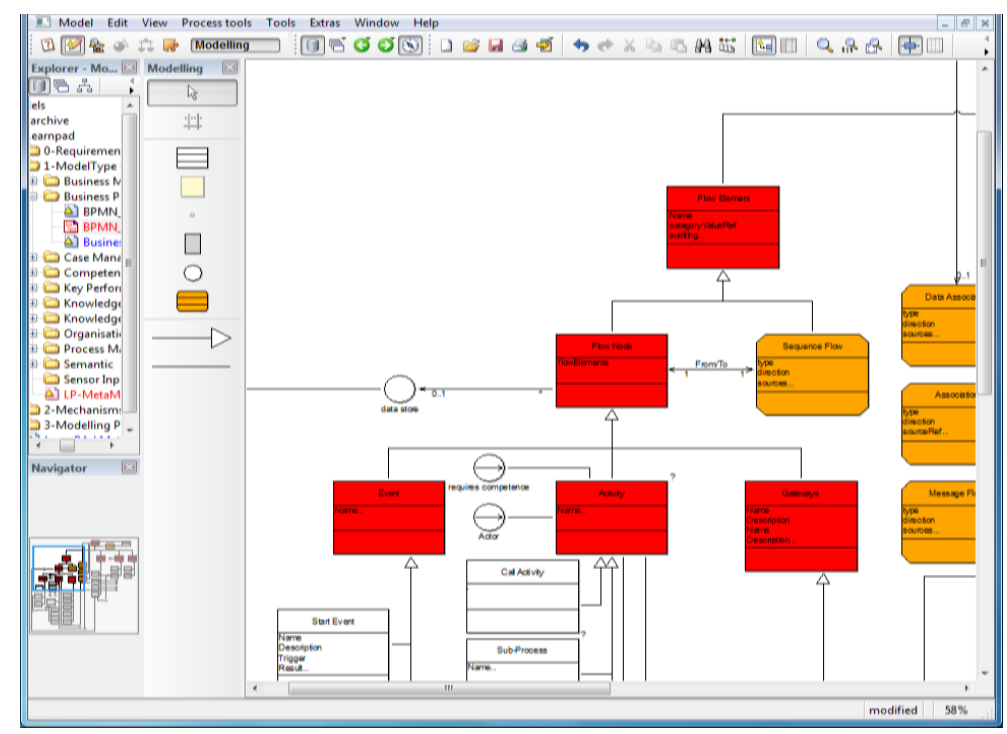

Figure 6 Meta-model Classes Model Type Sample

In order to specify proper graphical representation of each concept in the meta model as it is described in non-functional requirements, we specify another model type called "Graphical Notation Model Type"

Graphical Notation Model Type: in order to specify graphical representation of each concepts in meta-models according to non-functional this model type allows to describe graphical representation with assigning concrete images.

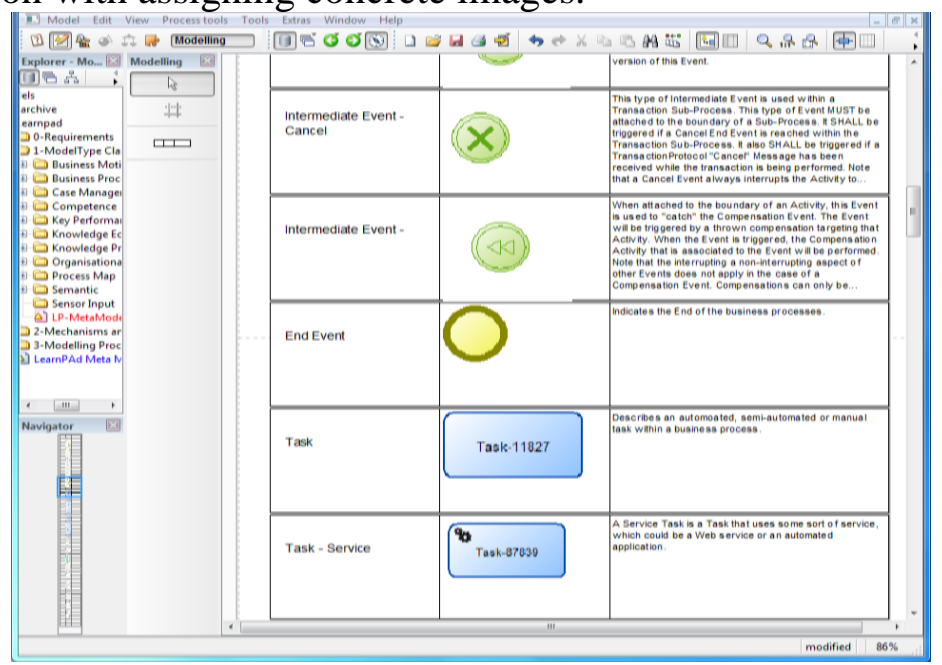

Figure 7Graphical Notation Model Type Sample

Having graphical notations for the concepts in UML suggested by OMG as initial graphical notation we improved the graphical notation via multi user test in several iterations and we suggested improvements such as differentiating representation of abstract classes, classes and relation classes from each other, dynamic representation of Pointer concept depending on which weaving type it is representing.

\section{Evaluation Results}

We consider that evaluation of prototypes that have been implemented based on specification reported by the Modelling Method Design Environment and comparison between specification and the prototype is an appropriate evaluation approach in order to understand the knowledge transformation capability of the Modelling Method Design Environment from modelling method designer to the developers. 
The Learn PAd Modelling Environment Prototype available to free download in [19] has been implemented on ADOxx ${ }^{\circledR}$ by two teams consist of 8 developers in two different organizations located in two different countries. The investigations and functionality test show us that the prototype covers all concepts specified in Learn PAd PSMM and identified functionalities reported using the design environment.

Moreover we studied requirement assessment report [20] issued by Learn PAd Project Consortium, which investigates the realization status of solutions that shall consider requirement identified at the beginning of project. According the report $95 \%$ of the requirements has been considered in the specification of Learn PAD Modelling Method and the solutions in the prototype has been implemented earlier that they have been planned to be implemented.

\section{Conclusion}

In this work, the challenges have been introduced that we faced during the conceptualization of Learn PAd Modelling Method. Subsequently we investigate a modelling method development framework and conceptualization process that guided us during the conceptualization of the Learn PAd Modelling Method. Moreover we introduced a modelling method design environment that we proposed and used within the project. Then we concentrated in the evaluation of the proposed design environment. Informal evaluation results indicated that that the idea of supporting conceptualization process with a design environment is essential and effective. The first prototype developed and utilized within the project provides significant support.

Given that it was first prototype and it proposes new concepts and extensions that are not foreseen in the well-known industry standards, we observed that some users especially the users used to use those standards- have difficulties to understand the semantics and the notation of new concepts as well as association among them in the big picture. The extensive specification and documentation of evolution of the Modelling Method Design Environment as well as evaluation of new prototypes is future works

\section{References}

[1] M. M. Brown and J. L. Brudney, "Learning organizations in the public sector? A study of police agencies employing information and technology to," Public administration review, 63(1), pp. 30-43, 2003.

[2] K. M. Wiig, "Knowledge management in public administration," Journal of knowledge management, 6(3), pp. 224-239, 2002.

[3] A. Riege and N. Lindsay, "Knowledge management in the public sector:stake-holder partnerships in the public policy development," Journal of knowledge management, 10(3), pp. 24-39, 2006.

[4] A. v. E. T. Boer, "Knowledge acquisition from sources of law in public administration," In Knowledge Engineering and Management by the Massess, pp. 4458, 2010.

[5] B. Niehaves and R. Plattfaut, "Collaborative business process management: status quo and quo vadis," Business Process Management Journal, 17(3), pp. 384-402, 2011.

[6] M. A. Dikotla, M. R. Mahlatji and L. A. Makgahlela, "Knowledge management for the improvement of service delivery in South Africa's municipalities," Journal of Public Administration, 49(3), pp. 847-859, 2014.

[7] Learn PAd Project Consortium, "The EU Project Learn PAd," 2014. [Online]. Available: http://www.learnpad.eu/. [Accessed May 2015]. 
[8] R. Woitsch, D. Burke and W. Utz, "Business Oriented Learn PAd Whitepaper," EUProject Learn PAd, 2015.

[9] D. Karagiannis and H. Kühn, "Metamodelling Platforms," in LNCS 2455, France, 2002.

[10] H. Kühn, "Methodenintegration im Business Engineering, PhD Thesis (in German)," University of Vienna, 2004.

[11] OMILAB, “OMILAB Life Cycle,” [Online]. [Accessed 1305 2015].

[12] Eclipse, "Eclipse Modeling Framework," [Online]. Available: https://www.eclipse.org/modeling/emf/. [Accessed 11 May 2015].

[13] ADOxx.org, “ADOxx,” [Online]. Available: http://www.adoxx.org/live/home. [Accessed 11 May 2015].

[14] MetaCase, "MetaEdit+," [Online]. Available: http://www.metacase.com/products.html. [Accessed 11 May 2015].

[15] MSDL, “AToM3,” [Online]. Available: http://atom3.cs.mcgill.ca/. [Accessed 11 May 2015].

[16] Institute for Software Integrated Systems, Vanderbilt University, "GME:Generic Modeling Environment," [Online]. Available:

http://www.isis.vanderbilt.edu/projects/gme/. [Accessed 11 May 2015].

[17] ADOxx.org, "Modelling Method Design Environment," 2014. [Online]. Available: http://www.adoxx.org/live/web/learnpad-developer-space/design-environment. [Accessed 12 May 2015].

[18] The Atlantic Systems Guild Ltd, "Volere requirements specification template".

[19] Learn PAd Project Consortium, "Learn PAd Developer Space," 2015. [Online]. Available: http://www.adoxx.org/live/web/learnpad-developer-space/learn-padmodelling-environment-prototype-v2.0. [Accessed 0106 2015].

[20] N. Efendioglu, "Requirements Assessment Report," The EU Project Learn PAd, 2015.

[21] G. A. Boyne, "Sources of public service improvement: A critical review and research agenda," Journal of public administration research and theory 13(3), pp. 367-394, 2003.

[22] D. Karagiannis, W. Utz, R. Woitsch and A. Leutgeb, "Business Processes and Rules An eGoverment Case Study," Spring Symposium Standford, AAAI, USA, 2008.

[23] I. Horrocks, P. F. Patel-Schneider, H. Boley, S. Tabet, B. Grosof and M. Dean, "Semantic Web Rule Language," 2004. [Online]. Available: http://www.w3.org/Submission/SWRL/. [Accessed 13 May 2015].

[24] A. Pierantonio, M. Albayrak, N. Efendioglu, K. Hinkelmann, J. Januskevicius, D. S. Gianni Rosa (UDA), S. T. arbara Thonssen (FHNW), R. Woitsch, A. Bertolino and A. Polini, "D3.2 Design and Initial Implementation of Metamodels for Describing Business Processes in Public Administrations," EU-Project Learn PAd, 2015.

[25] Learn PAd Consortium, "The EU Project Learn PAd," 2014. [Online]. Available: http://www.learnpad.eu/. [Accessed May 2015]. 